PROCEEDINGS OF THE

AMERICAN MATHEMATICAL SOCIETY

Volume 136, Number 8, August 2008, Pages 2921-2931

S 0002-9939(08)09365-9

Article electronically published on April 3, 2008

\title{
ON THE $H^{1}-L^{1}$ BOUNDEDNESS OF OPERATORS
}

\author{
STEFANO MEDA, PETER SJÖGREN, AND MARIA VALLARINO \\ (Communicated by Andreas Seeger)
}

\begin{abstract}
We prove that if $q$ is in $(1, \infty), Y$ is a Banach space, and $T$ is a linear operator defined on the space of finite linear combinations of $(1, q)$-atoms in $\mathbb{R}^{n}$ with the property that

$$
\sup \{\|T a\| Y: a \text { is a }(1, q) \text {-atom }\}<\infty,
$$

then $T$ admits a (unique) continuous extension to a bounded linear operator from $H^{1}\left(\mathbb{R}^{n}\right)$ to $Y$. We show that the same is true if we replace $(1, q)$-atoms by continuous $(1, \infty)$-atoms. This is known to be false for $(1, \infty)$-atoms.
\end{abstract}

\section{INTRODUCTION}

In a recent paper, M. Bownik 3 showed that there exists a linear functional $F$ defined on finite linear combinations of $(1, \infty)$-atoms in $\mathbb{R}^{n}$ with the property that

$$
\sup \{|F(a)|: a \text { is a }(1, \infty) \text {-atom }\}<\infty,
$$

but which does not admit a continuous extension to $H^{1}\left(\mathbb{R}^{n}\right)$. If $v$ is a fixed function in $L^{1}\left(\mathbb{R}^{n}\right) \backslash\{0\}$, then the operator $B$, defined on finite linear combinations of $(1, \infty)$ atoms by $B f=F(f) v$, satisfies

$$
\sup \left\{\|B a\|_{L^{1}\left(\mathbb{R}^{n}\right)}: a \text { is a }(1, \infty) \text {-atom }\right\}<\infty
$$

but does not admit an extension to a bounded operator from $H^{1}\left(\mathbb{R}^{n}\right)$ to $L^{1}\left(\mathbb{R}^{n}\right)$. This shows that the argument "the operator $T$ maps $(1, \infty)$-atoms uniformly into $L^{1}\left(\mathbb{R}^{n}\right)$, and hence it extends to a bounded operator from $H^{1}\left(\mathbb{R}^{n}\right)$ to $L^{1}\left(\mathbb{R}^{n}\right)$ " is fallacious.

Fortunately, if $T$ is a Calderón-Zygmund operator, then the uniform boundedness of $T$ on $(1, \infty)$-atoms implies the boundedness from $H^{1}\left(\mathbb{R}^{n}\right)$ to $L^{1}\left(\mathbb{R}^{n}\right)$ (see, for instance, [11, Ch. 7.3, Lemma 1], [2, Ch. 1.9], [7, Ch. III.7] and [8, Thm 6.7.1]).

The purpose of this paper is to show that the operator $B$ constructed above is, to a certain extent, pathological. Indeed, we prove that if $q$ is in $(1, \infty), Y$ is a Banach space, and $T$ is a linear operator defined on finite linear combinations of $(1, q)$-atoms in $\mathbb{R}^{n}$ with the property that

$$
\sup \left\{\|T a\|_{Y}: a \text { is a }(1, q) \text {-atom }\right\}<\infty,
$$

then $T$ admits a unique continuous extension to a bounded linear operator from $H^{1}\left(\mathbb{R}^{n}\right)$ to $Y$. The same conclusion holds if we assume that $T$ is a linear operator

Received by the editors June 18, 2007.

2000 Mathematics Subject Classification. Primary 42B30, 46A22.

Key words and phrases. BMO, atomic Hardy space, extension of operators.

This work was partially supported by the Progetto Cofinanziat "Analisi Armonica". 
on finite linear combinations of continuous $(1, \infty)$-atoms in $\mathbb{R}^{n}$ with the property that

$$
\sup \left\{\|T a\|_{Y}: a \text { is a continuous }(1, \infty) \text {-atom }\right\}<\infty .
$$

Note that this does not contradict Bownik's example. Indeed, the restriction of the operator $B$ to continuous $(1, \infty)$-atoms extends to a bounded operator $\widetilde{B}$ from $H^{1}\left(\mathbb{R}^{n}\right)$ to $L^{1}\left(\mathbb{R}^{n}\right)$. However, $B$ and $\widetilde{B}$ will agree on continuous $(1, \infty)$-atoms but not on all $(1, \infty)$-atoms.

To explain the idea of the proofs of these results, we need more notation. Suppose that $q$ is in $(1, \infty]$, and denote by $H_{\text {fin }}^{1, q}\left(\mathbb{R}^{n}\right)$ the vector space of all finite linear combinations of $(1, q)$-atoms. Notice that $H_{\text {fin }}^{1, q}\left(\mathbb{R}^{n}\right)$ consists of all $L^{q}\left(\mathbb{R}^{n}\right)$ functions with compact support and integral 0. Clearly, $H_{\text {fin }}^{1, q}\left(\mathbb{R}^{n}\right)$ is a dense subspace of $H^{1}\left(\mathbb{R}^{n}\right)$. We may define a norm on $H_{\text {fin }}^{1, q}\left(\mathbb{R}^{n}\right)$ as follows:

$$
\|f\|_{H_{\text {fin }}^{1, q}\left(\mathbb{R}^{n}\right)}=\inf \left\{\sum_{j=1}^{N}\left|\lambda_{j}\right|: f=\sum_{j=1}^{N} \lambda_{j} a_{j}, a_{j} \text { is a }(1, q) \text {-atom, } N \in \mathbb{N}\right\} .
$$

Obviously $\|f\|_{H^{1}\left(\mathbb{R}^{n}\right)} \leq\|f\|_{H_{\mathrm{fin}}^{1, q}\left(\mathbb{R}^{n}\right)}$ for every $f$ in $H_{\mathrm{fin}}^{1, q}\left(\mathbb{R}^{n}\right)$. An example due to Y. Meyer (see [12, p. 513], Bownik's paper [3] or [7, p. 370]) shows that $\|\cdot\|_{H^{1}\left(\mathbb{R}^{n}\right)}$ and $\|\cdot\|_{H_{\mathrm{fin}}^{1, \infty}\left(\mathbb{R}^{n}\right)}$ are inequivalent norms on $H_{\mathrm{fin}}^{1, \infty}\left(\mathbb{R}^{n}\right)$. This is the starting point of Bownik's construction.

We prove that Meyer's example itself is somewhat exceptional. Indeed, by using the maximal characterisation of $H^{1}\left(\mathbb{R}^{n}\right)$, we show that if $q<\infty$, then $\|\cdot\|_{H^{1}\left(\mathbb{R}^{n}\right)}$ and $\|\cdot\|_{H_{\text {fin }}^{1, q}\left(\mathbb{R}^{n}\right)}$ are equivalent norms on $H_{\text {fin }}^{1, q}\left(\mathbb{R}^{n}\right)$ (see Section [3). Similarly, we prove that $\|\cdot\|_{H^{1}\left(\mathbb{R}^{n}\right)}$ and $\|\cdot\|_{H_{\text {fin }}^{1, \infty}\left(\mathbb{R}^{n}\right)}$ are equivalent norms on $H_{\text {fin }}^{1, \infty}\left(\mathbb{R}^{n}\right) \cap C\left(\mathbb{R}^{n}\right)$. This immediately implies that operators defined on $H_{\text {fin }}^{1, q}\left(\mathbb{R}^{n}\right)$ which have either property (1.1) or property (1.2) automatically extend to bounded operators from $H^{1}\left(\mathbb{R}^{n}\right)$ to $L^{1}\left(\mathbb{R}^{n}\right)$.

As discussed briefly in Section 3, this equivalence of norms remains true for $H^{p}\left(\mathbb{R}^{n}\right)$ with $0<p<1$ and $(p, q)$-atoms.

The extension property for operators was also proved, by different methods, for $0<p \leq 1$ and $(p, 2)$-atoms and operators taking values in quasi-Banach spaces, by D. Yang and Y. Zhou [17].

A theory of Hardy spaces has been developed in spaces of homogeneous type; see R.R. Coifman and G. Weiss [5]. It is, however, not evident whether our results extend to this case in general. Nevertheless, let $M$ be such a space. By a simple functional analysis argument, we show that if $q$ is in $(1, \infty)$ and $T$ is an operator defined on $H_{\text {fin }}^{1, q}(M)$ satisfying the analogue of (1.1), then $T$ automatically extends to a bounded operator from $H^{1}(M)$ to $L^{1}(M)$ (see Section 4). It may be worth noticing that the proof of this result also applies to certain metric measured spaces $(M, \rho, \mu)$ where $\mu$ is only "locally doubling" [10], 4], and [16].

For so-called RD-spaces, which are spaces of homogeneous type having "dimension $n$ " in a certain sense, our complete results were recently extended in the paper [9] by L. Grafakos, L. Liu and Yang. These authors consider $n /(n+1)<p \leq 1$ and quasi-Banach-valued operators.

The authors wish to thank N. Th. Varopoulos for useful conversations on the subject of this paper. 


\section{Notation AND TERMinOLOGY}

Suppose that $(M, \rho, \mu)$ is a space of homogeneous type in the sense of Coifman and Weiss [5] and that $\mu$ is a $\sigma$-finite measure. For the sake of simplicity, we shall assume that $\mu(M)$ is infinite.

Suppose that $q$ is in $(1, \infty]$. For each closed ball $B$ in $M$, we denote by $L_{0}^{q}(B)$ the space of all functions in $L^{q}(M)$ which are supported in $B$ and have integral 0 . Clearly $L_{0}^{q}(B)$ is a closed subspace of $L^{q}(M)$. The union of all spaces $L_{0}^{q}(B)$ as $B$ varies over all balls coincides with the space $L_{c, 0}^{q}(M)$ of all functions in $L^{q}(M)$ with compact support and integral 0. Fix a reference point $o$ in $M$ and for each positive integer $k$ denote by $B_{k}$ the ball centred at $o$ with radius $k$. A convenient way of topologising $L_{c, 0}^{q}(M)$ is to interpret $L_{c, 0}^{q}(M)$ as the strict inductive limit of the spaces $L_{c, 0}^{q}\left(B_{k}\right)$ (see [1, II, p. 33] for the definition of the strict inductive limit topology). We denote by $X^{q}$ the space $L_{c, 0}^{q}(M)$ with this topology, and write $X_{k}^{q}$ for $L_{c, 0}^{q}\left(B_{k}\right)$.

We recall the basic definitions and results concerning the atomic Hardy space $H^{1}(M)$. The reader is referred to [5] and the references therein for this and more on Hardy spaces defined on spaces of homogeneous type. Suppose that $q$ is in $(1, \infty]$. A $(1, q)$-atom is a function $a$ in $L^{q}(M)$ supported in a ball $B$, with mean value 0 and such that

$$
\left(\frac{1}{\mu(B)} \int_{B}|a|^{q} \mathrm{~d} \mu\right)^{1 / q} \leq \mu(B)^{-1}
$$

if $q$ is finite, and $\|a\|_{\infty} \leq \mu(B)^{-1}$ if $q=\infty$. We denote by $H^{1, q}(M)$ the space of all functions $g$ in $L^{1}(M)$ which admit a decomposition of the form $g=\sum_{j} \lambda_{j} a_{j}$, where the $a_{j}$ are $(1, q)$-atoms and the $\lambda_{j}$ are complex numbers such that $\sum_{j}\left|\lambda_{j}\right|<$ $\infty$. The norm $\|g\|_{H^{1, q}}$ of $g$ in $H^{1, q}(M)$ is the infimum of $\sum_{j}\left|\lambda_{j}\right|$ over all such decompositions. It is well known that all the spaces $H^{1, q}(M)$ with $q \in(1, \infty)$ coincide with $H^{1, \infty}(M)$, and we denote them all by $H^{1}(M)$. Clearly, the vector space $H_{\text {fin }}^{1, q}(M)$ of all finite linear combinations of $(1, q)$-atoms is dense in $H^{1}(M)$ with respect to the norm of $H^{1}(M)$, for $q$ in $(1, \infty]$. Observe also that $H_{\text {fin }}^{1, q}(M)$ and $L_{c, 0}^{q}(M)$ agree as vector spaces, and so do the space of finite linear combinations of continuous $(1, \infty)$-atoms and $H_{\text {fin }}^{1, \infty}(M) \cap C\left(\mathbb{R}^{n}\right)$.

For each ball $B$ and each locally integrable function $f$, we denote by $f_{B}$ the average of $f$ on $B$. Recall that $B M O$ is the Banach space of all locally integrable functions $f$, defined modulo constants, such that

$$
\|f\|_{B M O}=\sup _{B} \frac{1}{\mu(B)} \int_{B}\left|f-f_{B}\right| \mathrm{d} \mu<\infty .
$$

The dual of $H^{1}(M)$ may be identified with $B M O$.

There are several characterisations of the space $H^{1}\left(\mathbb{R}^{n}\right)$. We shall make use of the so-called maximal characterisation, which we briefly recall. Suppose that $m$ is an integer with $m>n$, and denote by $\mathcal{A}_{m}$ the set of all functions $\varphi$ in the Schwartz space $\mathcal{S}\left(\mathbb{R}^{n}\right)$ such that

$$
\sup _{|\beta| \leq m} \sup _{x \in \mathbb{R}^{n}}(1+|x|)^{m}\left|D^{\beta} \varphi(x)\right| \leq 1,
$$

where $|\beta|$ denotes the length of the multi-index $\beta$. For $\varphi$ in $\mathcal{S}\left(\mathbb{R}^{n}\right)$ denote by $\varphi_{t}$ the function $t^{-n} \varphi(\cdot / t)$. Given $f$ in $L^{1}\left(\mathbb{R}^{n}\right)$, define the "grand maximal function" 
$\mathcal{M}_{m} f$ by

$$
\mathcal{M}_{m} f=\sup _{\varphi \in \mathcal{A}_{m}} \sup _{t>0}\left|\varphi_{t} * f\right| .
$$

The following result is classical [6], [13, 7], and [15].

Theorem 2.1. Suppose that $f$ is in $L^{1}\left(\mathbb{R}^{n}\right)$. The following are equivalent:

(i) $f$ is in $H^{1}\left(\mathbb{R}^{n}\right)$;

(ii) the grand maximal function $\mathcal{M}_{m} f$ is in $L^{1}\left(\mathbb{R}^{n}\right)$.

Furthermore, $f \mapsto\left\|\mathcal{M}_{m} f\right\|_{L^{1}\left(\mathbb{R}^{n}\right)}$ is an equivalent norm on $H^{1}\left(\mathbb{R}^{n}\right)$.

The letter $C$ will denote a positive constant, which need not be the same at different occurrences. Given two positive quantities $A$ and $B$, we shall mean by $A \sim B$ that there exists a constant $C$ such that $1 / C \leq A / B \leq C$.

\section{The EuClideAn CASE}

In this section we work in the classical setting of $\mathbb{R}^{n}$.

Theorem 3.1. The following hold:

(i) if $q<\infty$, then $\|\cdot\|_{H_{\mathrm{fin}}^{1, q}\left(\mathbb{R}^{n}\right)}$ and $\|\cdot\|_{H^{1}\left(\mathbb{R}^{n}\right)}$ are equivalent norms on $H_{\mathrm{fin}}^{1, q}\left(\mathbb{R}^{n}\right)$;

(ii) the two norms $\|\cdot\|_{H_{\mathrm{fin}}^{1, \infty}\left(\mathbb{R}^{n}\right)}$ and $\|\cdot\|_{H^{1}\left(\mathbb{R}^{n}\right)}$ are equivalent on $H_{\mathrm{fin}}^{1, \infty}\left(\mathbb{R}^{n}\right) \cap$ $C\left(\mathbb{R}^{n}\right)$.

Proof. Clearly, $\|f\|_{H^{1}\left(\mathbb{R}^{n}\right)} \leq\|f\|_{H_{\text {fin }}^{1, q}\left(\mathbb{R}^{n}\right)}$ for $f$ in $H_{\text {fin }}^{1, q}\left(\mathbb{R}^{n}\right)$ and for $q$ in $(1, \infty]$. Thus, we have to show that for every $q$ in $(1, \infty)$ there exists a constant $C$ such that

$$
\|f\|_{H_{\text {fin }}^{1, q}\left(\mathbb{R}^{n}\right)} \leq C\|f\|_{H^{1}\left(\mathbb{R}^{n}\right)} \quad \forall f \in H_{\text {fin }}^{1, q}\left(\mathbb{R}^{n}\right),
$$

and that a similar estimate holds for $q=\infty$ and all $f$ in $H_{\text {fin }}^{1, \infty}\left(\mathbb{R}^{n}\right) \cap C\left(\mathbb{R}^{n}\right)$.

Suppose that $q$ is in $(1, \infty]$ and that $f$ is in $H_{\text {fin }}^{1, q}\left(\mathbb{R}^{n}\right)$ with $\|f\|_{H^{1}\left(\mathbb{R}^{n}\right)}=1$. By the translation invariance of Lebesgue measure, we may assume that the support of $f$ is contained in the closed ball $B=B(0, R)$ centred at 0 with radius $R$. For each $k$ in $\mathbb{Z}$, denote by $\Omega_{k}$ the level set $\left\{x \in \mathbb{R}^{n}: \mathcal{M}_{m} f(x)>2^{k}\right\}$ of the grand maximal function $\mathcal{M}_{m} f$ of $f$. We choose Whitney cubes $Q_{i}^{k}, i \in \mathbb{N}$, with disjoint interiors satisfying $\Omega_{k}=\bigcup_{i} Q_{i}^{k}$ and

$$
\operatorname{diam}\left(Q_{i}^{k}\right) \leq \eta \operatorname{dist}\left(Q_{i}^{k}, \Omega_{k}^{c}\right) \leq 4 \operatorname{diam}\left(Q_{i}^{k}\right)
$$

where $\eta$ is a suitable constant in $(0,1)$. Except for the factor $\eta$, this is Theorem VI.1 of [14, p. 167]. The only modification needed in the proof of [14 concerns the choice of the constant denoted $c$.

By following closely the proof of [15, Theorem III.2, p. 107] or [13, Theorem 3.5, pp. 12-18], we produce an atomic decomposition of $f$ of the form

$$
f=\sum_{i, k} \lambda_{i}^{k} a_{i}^{k}
$$

such that the following hold:

(a) $\left|\lambda_{i}^{k} a_{i}^{k}\right| \leq C 2^{k}$ for every $k$ in $\mathbb{Z}$;

(b) for each $k$ in $\mathbb{Z}$, the atoms $a_{i}^{k}$ are supported in balls $B_{i}^{k}$ concentric with the $Q_{i}^{k}$ and contained in $\Omega_{k}$. By choosing the constant $\eta$ in (3.1) small enough, depending on the dimension, we can also ensure that the family $\left\{B_{i}^{k}\right\}_{i}$ has the bounded overlap property, uniformly with respect to $k$; 
(c) there exists a constant $C$ independent of $f$ such that

$$
\sum_{i, k}\left|\lambda_{i}^{k}\right| \leq C\|f\|_{H^{1}\left(\mathbb{R}^{n}\right)}=C .
$$

We write $2 B$ for the closed ball concentric with $B$ whose radius is twice as large. For $\varphi$ in $\mathcal{A}_{m}$ and $x$ in $\mathbb{R}^{n} \backslash(2 B)$ one then has

$$
\begin{aligned}
\left|\varphi_{t} * f(x)\right| & \leq t^{-n} \sup _{y \in B^{c}}|\varphi(y / t)|\|f\|_{L^{1}\left(\mathbb{R}^{n}\right)} \\
& \leq t^{-n}(1+R / t)^{-m}\|f\|_{L^{1}\left(\mathbb{R}^{n}\right)} \quad \forall t \in \mathbb{R}^{+}
\end{aligned}
$$

so that

$$
\mathcal{M}_{m} f(x)=\sup _{\varphi \in \mathcal{A}_{m}} \sup _{t>R}\left|\varphi_{t} * f(x)\right| \leq R^{-n}
$$

since $m>n$. Now, if $x$ is in $\Omega_{k} \backslash(2 B)$, the above inequality and the definition of $\Omega_{k}$ force $2^{k}<R^{-n}$; denote by $k^{\prime}$ the largest integer $k$ such that $2^{k}<R^{-n}$. Then $\overline{\Omega_{k}}$ is contained in $2 B$ for $k>k^{\prime}$.

Next we define the functions $h$ and $\ell$ by

$$
h=\sum_{k \leq k^{\prime}} \sum_{i} \lambda_{i}^{k} a_{i}^{k} \quad \text { and } \quad \ell=\sum_{k>k^{\prime}} \sum_{i} \lambda_{i}^{k} a_{i}^{k} .
$$

Observe that both these series converge in $L^{1}\left(\mathbb{R}^{n}\right)$, simply because $\sum_{i, k}\left|\lambda_{i}^{k}\right|<\infty$, so that $h$ and $\ell$ have integral 0. Clearly, $f=h+\ell$. Furthermore, the support of $\ell$ is contained in $2 B$, because it is contained in $\bar{\Omega}_{k}$ by (b) above, and $\bar{\Omega}_{k}$ is contained in $2 B$ for all $k>k^{\prime}$. Therefore $h=f=0$ in $(2 B)^{c}$.

To estimate the size of $h$ in $2 B$, we use (a) above and the bounded overlap property of (b), getting

$$
|h| \leq C \sum_{k \leq k^{\prime}} 2^{k} \leq C 2^{k^{\prime}} \leq C|2 B|^{-1} .
$$

This proves that $h / C$ is a $(1, \infty)$-atom, where $C$ is independent of $f$.

Now we assume that $q<\infty$ and conclude the proof of (i). Observe that $\ell$ is in $L^{q}\left(\mathbb{R}^{n}\right)$, because $\ell=f-h$, and both $f$ and $h$ are in $L^{q}\left(\mathbb{R}^{n}\right)$.

We claim that the series $\sum_{k>k^{\prime}} \sum_{i} \lambda_{i}^{k} a_{i}^{k}$ converges to $\ell$ in $L^{q}\left(\mathbb{R}^{n}\right)$.

Fixing $s$ in $\mathbb{Z}$, we shall estimate $\sum_{k>k^{\prime}} \sum_{i}\left|\lambda_{i}^{k} a_{i}^{k}\right|$ in $\Omega_{s} \backslash \Omega_{s+1}$. First observe that all terms with $k>s$ vanish outside $\Omega_{s+1}$. Then apply (a) and (b) to get the pointwise bound

$$
\sum_{k>k^{\prime}} \sum_{i}\left|\lambda_{i}^{k} a_{i}^{k}\right| \leq C \sum_{k \leq s} 2^{k} \leq C 2^{s} \leq C \mathcal{M}_{m} f .
$$

The constants $C$ above are independent of $f$ and $s$, so that

$$
\sum_{k>k^{\prime}} \sum_{i}\left|\lambda_{i}^{k} a_{i}^{k}\right| \leq C \mathcal{M}_{m} f
$$

in all of $\mathbb{R}^{n}$, with $C$ independent of $f$. Note that $\mathcal{M}_{m} f$ is in $L^{q}\left(\mathbb{R}^{n}\right)$, since $f$ is. This implies that the series defining $\ell$ converges almost everywhere and the limit must coincide with the $L^{1}$ limit $\ell$. The Lebesgue dominated convergence theorem now implies that $\sum_{k>k^{\prime}} \sum_{i} \lambda_{i}^{k} a_{i}^{k}$ converges to $\ell$ in $L^{q}\left(\mathbb{R}^{n}\right)$, and the claim is proved.

Finally, for each positive integer $N$ we denote by $F_{N}$ the finite set of all pairs of integers $(i, k)$ such that $k>k^{\prime}$ and $|i|+|k| \leq N$, and by $\ell_{N}$ the function $\sum_{(i, k) \in F_{N}} \lambda_{i}^{k} a_{i}^{k}$. The function $\ell_{N}$ is in $H_{\mathrm{fin}}^{1, q}\left(\mathbb{R}^{n}\right)$, and $f=h+\ell_{N}+\left(\ell-\ell_{N}\right)$. 
Observe that $\ell-\ell_{N}$ will be a small multiple of a $(1, q)$-atom for large $N$. Indeed, by taking $N$ large enough, we can make the corresponding coefficient less than any given $\varepsilon$ in $\mathbb{R}^{+}$. Then

$$
\|f\|_{H_{\mathrm{fin}}^{1, q}\left(\mathbb{R}^{n}\right)} \leq C+\sum_{(i, k) \in F_{N}}\left|\lambda_{i}^{k}\right|+\varepsilon,
$$

so that

$$
\|f\|_{H_{\mathrm{fin}}^{1, q}\left(\mathbb{R}^{n}\right)} \leq C+\sum_{(i, k) \in F_{N}}\left|\lambda_{i}^{k}\right| \leq C,
$$

by property (c) above, as required to conclude the proof of (i).

Now we finish the proof of (ii). Assume that $f$ is a continuous function in $H_{\mathrm{fin}}^{1, \infty}\left(\mathbb{R}^{n}\right)$. A careful examination of the proof of [15. Theorem III.2, pp. 1078] or [13. Theorem 3.5, pp. 12-18] shows that the atoms $a_{i}^{k}$ that appear in the decomposition (3.2) are then continuous. Furthermore, we see that for each $k$ and $i$ the function $\lambda_{i}^{k} a_{i}^{k}$ depends only on the restriction of $f$ to a ball $\tilde{B}_{i}^{k}$ which is a concentric enlargement of the ball $B_{i}^{k}$ from (b) above, by a fixed scaling factor. It is straightforward to check that if $f$ is constant in $\tilde{B}_{i}^{k}$, then $\lambda_{i}^{k} a_{i}^{k}=0$ and that there exists an absolute constant $C$ such that if $|f|<\varepsilon$ in $\tilde{B}_{i}^{k}$, then $\left|\lambda_{i}^{k} a_{i}^{k}\right|<C \varepsilon$.

Since trivially $\mathcal{M}_{m} f \leq C_{n}\|f\|_{\infty}$, where the constant $C_{n}$ depends only on $n$, the level set $\Omega_{k}$ is empty for all $k$ such that $2^{k} \geq C_{n}\|f\|_{\infty}$. We denote by $k^{\prime \prime}$ the largest integer for which the last inequality does not hold. Then the index $k$ in the sum defining $\ell$ in (3.3) will run only over $k^{\prime}<k \leq k^{\prime \prime}$.

Let $\varepsilon$ be positive. Since $f$ is uniformly continuous, there exists a positive $\delta$ such that $|x-y|<\delta$ implies

$$
|f(x)-f(y)|<\varepsilon \text {. }
$$

Write $\ell=\ell_{1}^{\varepsilon}+\ell_{2}^{\varepsilon}$ with

$$
\ell_{1}^{\varepsilon}=\sum_{(i, k) \in F_{1}} \lambda_{i}^{k} a_{i}^{k} \quad \text { and } \quad \ell_{2}^{\varepsilon}=\sum_{(i, k) \in F_{2}} \lambda_{i}^{k} a_{i}^{k},
$$

where $F_{1}=\left\{(i, k): \operatorname{diam}\left(\tilde{B}_{i}^{k}\right) \geq \delta, k^{\prime}<k \leq k^{\prime \prime}\right\}$ and $F_{2}=\left\{(i, k): \operatorname{diam}\left(\tilde{B}_{i}^{k}\right)<\right.$ $\left.\delta, k^{\prime}<k \leq k^{\prime \prime}\right\}$. Since $F_{1}$ is a finite set, $\ell_{1}^{\varepsilon}$ is continuous.

To estimate $\ell_{2}^{\varepsilon}$, we denote by $x_{i}^{k}$ the centre of the ball $B_{i}^{k}$ and write for $(i, k)$ in $F_{2}$

$$
f(x)=f\left(x_{i}^{k}\right)+f(x)-f\left(x_{i}^{k}\right) .
$$

Then $\left|\lambda_{i}^{k} a_{i}^{k}\right|<C \varepsilon$, because $\left|f(x)-f\left(x_{i}^{k}\right)\right|<\varepsilon$ for $x$ in $\tilde{B}_{i}^{k}$. For fixed $k$ the balls $\left\{B_{i}^{k}\right\}_{i}$ have uniformly bounded overlap, so there exists an absolute constant $C$ such that

$$
\left|\ell_{2}^{\varepsilon}\right| \leq C \sum_{k^{\prime}<k \leq k^{\prime \prime}} \varepsilon \leq C\left(k^{\prime \prime}-k^{\prime}\right) \varepsilon
$$

Since $\varepsilon$ is arbitrary, we can thus split $\ell$ into a continuous part and a part that is uniformly arbitarily small. It follows that $\ell$ is continuous. But then $h=f-\ell$ is also continuous, so that $h$ is a continuous $(1, \infty)$-atom, multiplied by a factor $C$.

To find a finite atomic decomposition of $\ell$, we again use the splitting $\ell=\ell_{1}^{\varepsilon}+\ell_{2}^{\varepsilon}$. Clearly $\ell_{1}^{\varepsilon}$ is for each $\varepsilon$ a finite linear combination of continuous $(1, \infty)$-atoms, and the $\ell^{1}$ norm of the coefficients is controlled by $\|f\|_{H^{1}}$, in view of (c). Observe that $\ell_{2}^{\varepsilon}=\ell-\ell_{1}^{\varepsilon}$ is continuous. Further, $\ell_{2}^{\varepsilon}$ is supported in $2 B$, has integral 0 and satisfies $\left|\ell_{2}^{\varepsilon}\right| \leq C\left(k^{\prime \prime}-k^{\prime}\right) \varepsilon$. Choosing $\varepsilon$, we can thus make $\ell_{2}^{\varepsilon}$ into an arbitrarily small multiple of a continuous $(1, \infty)$-atom. 
To sum up, $f=h+\ell_{1}^{\varepsilon}+\ell_{2}^{\varepsilon}$ gives the desired finite atomic decomposition of $f$, with coefficients controlled by $\|f\|_{H^{1}}$.

We have completed the proof of (ii) and that of the theorem.

Remark 3.2. Theorem 3.1 (ii) implies that any function $f$ in $H_{\text {fin }}^{1, \infty}\left(\mathbb{R}^{n}\right) \cap C\left(\mathbb{R}^{n}\right)$ admits a finite decomposition in $(1, \infty)$-atoms such that the sum of the corresponding coefficients is $\leq C\|f\|_{H^{1}\left(\mathbb{R}^{n}\right)}$. Actually, the proof of Theorem 3.1 (ii) shows that we can construct this finite decomposition in such a way that it involves only continuous $(1, \infty)$-atoms.

Remark 3.3. Theorem 3.1 extends to $H^{p}\left(\mathbb{R}^{n}\right)$ with $0<p<1$ and $(p, q)$-atoms, where one can now have $1 \leq q \leq \infty$. The proof is rather similar to the one given above, so we only briefly describe the modifications needed for part (i). Thus let $1 \leq q<\infty$. Given $f \in H_{\text {fin }}^{p, q}\left(\mathbb{R}^{n}\right)$ supported in a ball $B_{R}$, the first step is the inequality $\mathcal{M}_{m} f \leq C R^{-n / p}\|f\|_{H^{p}\left(\mathbb{R}^{n}\right)}$, valid outside a larger ball $B_{C R}$. One proves this by comparing the values of $\mathcal{M}_{m} f$ at different points and using the fact that $\left\|\mathcal{M}_{m} f\right\|_{L^{p}\left(\mathbb{R}^{n}\right)} \sim\|f\|_{H^{p}\left(\mathbb{R}^{n}\right)}$. Then the $\Omega_{k}$ and the decompositions $f=\sum \lambda_{i}^{k} a_{i}^{k}=$ $h+\ell$ are introduced as above. The sum $\ell$ now converges in $\mathcal{S}^{\prime}$ and is dominated by $\mathcal{M}_{m} f$. If $q>1$, we have $\mathcal{M}_{m} f \in L^{q}\left(\mathbb{R}^{n}\right)$ and conclude as before that $\ell$ converges in $L^{q}\left(\mathbb{R}^{n}\right)$. For $q=1$, the tail sum $S_{\kappa}=\sum_{k \geq \kappa} \sum_{i} \lambda_{i}^{k} a_{i}^{k}$ tends to 0 in $L^{1}\left(\mathbb{R}^{n}\right)$ as $\kappa \rightarrow+\infty$, because $S_{\kappa}$ is nonzero only in $\Omega_{\kappa}$ and not larger than $|f|+C 2^{\kappa}$ there, and $\left|\Omega_{\kappa}\right|=o\left(2^{-\kappa}\right)$ as $\kappa \rightarrow+\infty$. The rest of the proof proceeds as before. See also [9. Theorem 5.6].

Corollary 3.4. Suppose that $Y$ is a Banach space and that one of the following holds:

(i) $q$ is in $(1, \infty)$ and $T: H_{\text {fin }}^{1, q}\left(\mathbb{R}^{n}\right) \rightarrow Y$ is a linear operator such that

$$
A:=\sup \left\{\|T a\|_{Y}: a \text { is a }(1, q) \text {-atom }\right\}<\infty ;
$$

(ii) $T$ is a $Y$-valued linear operator defined on continuous $(1, \infty)$-atoms such that

$$
A:=\sup \left\{\|T a\|_{Y}: a \text { is a continuous }(1, \infty) \text {-atom }\right\}<\infty .
$$

Then there exists a unique bounded linear operator $\widetilde{T}$ from $H^{1}\left(\mathbb{R}^{n}\right)$ to $Y$ which extends $T$.

Proof. We consider the case (i). Suppose that $f$ is in $H_{\text {fin }}^{1, q}\left(\mathbb{R}^{n}\right), f=\sum_{j=1}^{N} \lambda_{j} a_{j}$ say, where $a_{j}$ are $(1, q)$-atoms. Then the assumption and the triangle inequality give

$$
\|T f\|_{Y} \leq A \sum_{j=1}^{N}\left|\lambda_{j}\right| .
$$

By taking the infimum of the right-hand side with respect to all decompositions of $f$ as a finite sum of $(1, q)$-atoms, we obtain

$$
\|T f\|_{Y} \leq A\|f\|_{H_{\text {fin }}^{1, q}\left(\mathbb{R}^{n}\right)} .
$$

Now, Theorem 3.1(i) implies that the right-hand side is dominated by $C A\|f\|_{H^{1}\left(\mathbb{R}^{n}\right)}$, where $C$ does not depend on $f$, and a density argument completes the proof of the corollary.

The case (ii) is similar. 
Remark 3.5. The statement of Corollary 3.4 (i) becomes false if we replace $q$ by $\infty$. A counterexample is given by the operator $B$ defined in the Introduction. Note also that Corollary 3.4 applies to linear functionals.

\section{Results on spaces of homogeneous type}

In this section, we work in a space of homogeneous type $(M, \rho, \mu)$. Recall that we assume that $\mu$ is $\sigma$-finite and that $\mu(M)$ is infinite.

Theorem 4.1. Suppose that $q$ is in $(1, \infty)$ and that $T$ is a linear operator defined on $H_{\text {fin }}^{1, q}(M)$ with the property that

$$
A:=\sup \left\{\|T a\|_{L^{1}(M)}: \text { a is a }(1, q) \text {-atom }\right\}<\infty .
$$

Then there exists a unique bounded linear operator $\widetilde{T}$ from $H^{1}(M)$ to $L^{1}(M)$ which extends $T$.

Proof. We prove the result in the case where $q=2$. The proof in the other cases is similar.

Suppose that $B$ is a ball. For each $f$ in $L_{0}^{2}(B)$ such that $\|f\|_{L^{2}(M)}=1$, the function $\mu(B)^{-1 / 2} f$ is a $(1,2)$-atom, so that

$$
\|T f\|_{L^{1}(M)} \leq A \mu(B)^{1 / 2} \quad \forall f \in L_{0}^{2}(B)
$$

by the assumption. In particular, the restriction of $T$ to $X_{k}^{2}$ is bounded from $X_{k}^{2}$ to $L^{1}(M)$ for each $k$. Thus, $T$ is bounded from $X^{2}$ to $L^{1}(M)$. It follows that $T^{*}$ is bounded from $L^{\infty}(M)$ to the dual of $X^{2}$. But the dual of $X^{2}$ is the quotient space $L_{\text {loc }}^{2}(M) / \mathbb{C}$, since that of $L_{c, 0}^{2}\left(B_{k}\right)$ is $L^{2}\left(B_{k}\right) / \mathbb{C}$. Now, for every $f$ in $L^{\infty}(M)$ and for every $(1,2)$-atom $a$,

$$
\langle T a, f\rangle=\left\langle a, T^{*} f\right\rangle=\int_{M} a T^{*} f \mathrm{~d} \mu,
$$

so that

$$
\left|\int_{M} a T^{*} f \mathrm{~d} \mu\right|=|\langle T a, f\rangle| \leq A\|f\|_{\infty} .
$$

A standard argument then shows that $T^{*} f$ belongs to $B M O(M)$ and that

$$
\left\|T^{*} f\right\|_{B M O(M)} \leq 2 A\|f\|_{\infty} \quad \forall f \in L^{\infty}(M) .
$$

We give the details for the reader's convenience. Suppose that $B$ is a ball and observe that

$$
\left[\int_{B}\left|T^{*} f-\left(T^{*} f\right)_{B}\right|^{2} \mathrm{~d} \mu\right]^{1 / 2}=\sup _{\|\varphi\|_{L^{2}(B)}=1}\left|\int_{B} \varphi\left(T^{*} f-\left(T^{*} f\right)_{B}\right) \mathrm{d} \mu\right| .
$$

But

$$
\begin{aligned}
\int_{B} \varphi\left(T^{*} f-\left(T^{*} f\right)_{B}\right) \mathrm{d} \mu & =\int_{B}\left(\varphi-\varphi_{B}\right)\left(T^{*} f-\left(T^{*} f\right)_{B}\right) \mathrm{d} \mu \\
& =\int_{B}\left(\varphi-\varphi_{B}\right) T^{*} f \mathrm{~d} \mu,
\end{aligned}
$$

and since $\|\varphi\|_{L^{2}(B)}=1$,

$$
\left|\varphi_{B}\right| \leq\left[\frac{1}{\mu(B)} \int_{B}|\varphi|^{2} \mathrm{~d} \mu\right]^{1 / 2} \leq \mu(B)^{-1 / 2} .
$$


Write $\psi$ instead of $\varphi-\varphi_{B}$. Then

$$
\|\psi\|_{L^{2}(B)} \leq\|\varphi\|_{L^{2}(B)}+\left|\varphi_{B}\right| \mu(B)^{1 / 2} \leq 2,
$$

so that $\psi /\left(2 \mu(B)^{1 / 2}\right)$ is a $(1,2)$-atom. Therefore

$$
\left|\int_{B} \psi T^{*} f \mathrm{~d} \mu\right| \leq 2 A \mu(B)^{1 / 2}\|f\|_{\infty} .
$$

Combining the above, we conclude that for every ball $B$

$$
\left[\frac{1}{\mu(B)} \int_{B}\left|T^{*} f-\left(T^{*} f\right)_{B}\right|^{2} \mathrm{~d} \mu\right]^{1 / 2} \leq 2 A\|f\|_{\infty}
$$

and (4.1) follows.

Now we show that $T$ extends to a bounded operator from $H^{1}(M)$ to $L^{1}(M)$ with norm at most $2 A$. Observe that $X^{2}$ and $H_{\text {fin }}^{1,2}(M)$ coincide as vector spaces. For every $g$ in $H_{\text {fin }}^{1,2}(M)$ and for every $f$ in $L^{\infty}(M)$

$$
\begin{aligned}
|\langle T g, f\rangle| & =\left|\left\langle g, T^{*} f\right\rangle\right| \\
& \leq\|g\|_{H^{1}(M)}\left\|T^{*} f\right\|_{B M O(M)} \\
& \leq 2 A\|g\|_{H^{1}(M)}\|f\|_{L^{\infty}(M)} .
\end{aligned}
$$

By taking the supremum of both sides over all functions $f$ in $L^{\infty}(M)$ with $\|f\|_{L^{\infty}(M)}$ $=1$, we obtain that

$$
\|T g\|_{L^{1}(M)} \leq 2 A\|g\|_{H^{1}(M)} \quad \forall g \in H_{\text {fin }}^{1,2}(M) .
$$

Finally we observe that $H_{\text {fin }}^{1,2}(M)$ is dense in $H^{1}(M)$ (with respect to the norm of $H^{1}(M)$ ), and the required conclusion follows by a density argument.

Quite often one encounters the following situation. Suppose that $T$ is a bounded linear operator on $L^{2}(M)$. Then $T$ is automatically defined on $H_{\text {fin }}^{1,2}(M)$. Assume that

$$
A:=\sup \left\{\|T a\|_{L^{1}(M)}: a \text { is a }(1,2) \text {-atom }\right\}<\infty .
$$

By the previous result, the restriction of $T$ to $H_{\text {fin }}^{1,2}(M)$ has a unique extension to a bounded linear operator $\widetilde{T}$ from $H^{1}(M)$ to $L^{1}(M)$. The question is whether the operators $T$ and $\widetilde{T}$ are consistent, i.e., whether they coincide on the intersection $H^{1}(M) \cap L^{2}(M)$ of their domains. The answer to this question is in the affirmative, as the following proposition shows.

Proposition 4.2. Suppose that $T$ is bounded on $L^{2}(M)$ and that

$$
A:=\sup \left\{\|T a\|_{L^{1}(M)}: a \text { is a }(1,2) \text {-atom }\right\}<\infty .
$$

Denote by $\widetilde{T}$ the unique continuous linear extension of the restriction of $T$ to $H_{\text {fin }}^{1,2}(M)$ to an operator from $H^{1}(M)$ to $L^{1}(M)$. Then the operators $T$ and $\widetilde{T}$ agree on $H^{1}(M) \cap L^{2}(M)$.

Proof. Suppose that $f$ is in $L^{2}(M) \cap L^{\infty}(M)$ and that $g$ is in $L_{c, 0}^{2}(M)$. Denote by $T^{*}$ the transpose operator of $T$ (as an operator on $L^{2}(M)$ ). Then

$$
\int_{M} g T^{*} f \mathrm{~d} \mu=\int_{M} T g f \mathrm{~d} \mu .
$$


Since $g$ is in $H_{\text {fin }}^{1,2}(M)$ and the operators $T$ and $\widetilde{T}$ agree on $H_{\text {fin }}^{1,2}(M)$, we see that

$$
\begin{aligned}
\int_{M} T g f \mathrm{~d} \mu & =\int_{M} \widetilde{T} g f \mathrm{~d} \mu \\
& =\left\langle g,(\widetilde{T})^{*} f\right\rangle,
\end{aligned}
$$

where $(\widetilde{T})^{*}$ denotes the transpose of the operator $\widetilde{T}$ from $H^{1}(M)$ to $L^{1}(M)$. Note that $(\widetilde{T})^{*} f$ is in $B M O(M)$ and $g$ is a multiple of an atom. Thus the above scalar product $\left\langle g,(\widetilde{T})^{*} f\right\rangle$ (with respect to the duality between $H^{1}(M)$ and $B M O(M)$ ) may be written as $\int_{M} g(\widetilde{T})^{*} f \mathrm{~d} \mu$. Therefore, (4.2) and (4.3) imply that

$$
\int_{M} g\left[T^{*} f-(\widetilde{T})^{*} f\right] \mathrm{d} \mu=0 \quad \forall g \in L_{c, 0}^{2}(M),
$$

i.e., for all $g$ in $X^{2}$. Therefore $T^{*} f-(\widetilde{T})^{*} f=0$ in the dual space of $X^{2}$, i.e., in $L_{\text {loc }}^{2}(M) / \mathbb{C}$. This implies that $T^{*} f-(\widetilde{T})^{*} f$ is constant.

Now, suppose that $g$ is in $H^{1}(M) \cap L^{2}(M)$ and that $f$ is in $L^{2}(M) \cap L^{\infty}(M)$. Then

$$
\begin{aligned}
\int_{M} T g f \mathrm{~d} \mu & =\int_{M} g T^{*} f \mathrm{~d} \mu \\
& =\int_{M} g(\widetilde{T})^{*} f \mathrm{~d} \mu \\
& =\int_{M} \widetilde{T} g f \mathrm{~d} \mu .
\end{aligned}
$$

Since $f$ is an arbitrary function in $L^{2}(M) \cap L^{\infty}(M), T g-\widetilde{T} g=0$ almost everywhere, as required.

\section{REFERENCES}

1. N. Bourbaki, Topological Vector Spaces. Chapters 1-5, Elements of Mathematics, SpringerVerlag, Berlin, Heidelberg, New York, 1987. MR910295 (88g:46002)

2. M. Bownik, Anisotropic Hardy spaces and wavelets, Mem. Amer. Math. Soc. 164 (2003), vi+122 pp. MR.1982689 (2004e:42023)

3. M. Bownik, Boundedness of operators on Hardy spaces via atomic decompositions, Proc. Amer. Math. Soc. 133 (2005), 3535-3542. MR2163588 (2006d:42028)

4. A. Carbonaro, G. Mauceri, S. Meda, $H^{1}, B M O$ and singular integrals for certain measured metric spaces, submitted.

5. R. R. Coifman, G. Weiss, Extensions of Hardy spaces and their use in analysis, Bull. Amer. Math. Soc. 83 (1977), 569-645. MR0447954 (56:6264)

6. G. B. Folland, E. M. Stein, Hardy spaces on homogeneous groups, Princeton University Press, 1982. MR657581 (84h:43027)

7. J. García-Cuerva, J. L. Rubio de Francia, Weighted norm inequalities and related topics, North-Holland, 1985. MR807149 (87d:42023)

8. L. Grafakos, Classical and Modern Fourier Analysis, Pearson, 2004.

9. L. Grafakos, L. Liu, D. Yang, Maximal function characterizations of Hardy spaces on RDspaces and their applications, submitted.

10. G. Mauceri, S. Meda, BMO and $H^{1}$ for the Ornstein-Uhlenbeck operator, J. Funct. Anal. 252 (2007), 278-313. MR2357358

11. Y. Meyer, R. R. Coifman, Wavelets. Calderón-Zygmund and multilinear operators, Cambridge University Press, Cambridge, 1997. MR 1456993 (98e:42001)

12. Y. Meyer, M. H. Taibleson, G. Weiss, Some functional analytic properties of the spaces $B_{q}$ generated by blocks, Indiana Univ. Math. J. 34 (1985), 493-515. MR794574 (87c:46036) 
13. P. Sjögren, Lectures on atomic $H^{p}$ spaces theory in $\mathbb{R}^{n}$, Lecture Notes, University of Umeå, n. 5, 1981. See also www.chalmers.se/math/SV/kontakt/personal/larare-och-forskare/sjogrenpeter.

14. E. M. Stein, Singular integrals and differentiability properties of functions, Princeton University Press, 1970. MR0290095 (44:7280)

15. E. M. Stein, Harmonic analysis. Real variable methods, orthogonality and oscillatory integrals, Princeton Math. Series, No. 43, Princeton, NJ, 1993. MR1232192 (95c:42002)

16. M. Vallarino, Spaces $H^{1}$ and BMO on $a x+b$-groups, submitted.

17. D. Yang, Y. Zhou, A boundedness criterion via atoms for linear operators in Hardy spaces, to appear in Constr. Approx.

Dipartimento di Matematica e Applicazioni, Universitá degli Studi di MilanoBicocca, Via Cozzi, 53, 20125 Milano, Italy

E-mail address: stefano.meda@unimib.it

Department of Mathematical Sciences, University of Gothenburg, SE-412 96 Göteborg, Sweden; and Department of Mathematical Sciences, Chalmers University of TechNOLOGY, SE-412 96 GÖTEBorg, Sweden

E-mail address: peters@math.chalmers.se

Laboratoire Mapmo UMr 6628, Fédération Denis Poisson, Université d'Orléans, UfR Sciences, BÂtiment de mathématiques - Route de Chartres, B.P. 6759 - 45067 Orléans CEDEX 2, France

E-mail address: maria.vallarino@unimib.it 